\section{O agente comunitário de saúde no controle da tuberculose: conhecimentos e percepções}

\author{
Community health workers and tuberculosis \\ control: knowledge and perceptions
}

\footnotetext{
${ }^{1}$ Centro de Ciências da Saúde, Universidade Federal do Espírito Santo, Vitória, Brasil.

Correspondência E. L. N. Maciel Núcleo de Doenças Infecciosas, Centro de Ciências da Saúde, Universidade Federal do Espirito Santo. Av. Marechal Campos 1468, Vitória, ES 29040-090, Brasil. emaciel@ndi.ufes.br
}

\begin{abstract}
This study analyzes the knowledge of community health workers in tuberculosis control and their self-perceived knowledge and the importance they ascribe to their role in fighting the disease, in Vitória, Espírito Santo State, Brasil. The study adopted a cross-sectional design, with a pre-tested, semi-structured questionnaire completed by 105 randomly selected health workers. The comparison of proportions between the groups formed by stratification according to time-on-the-job used the chi-squared test with 5\% significance. Mean age was 34.5 ( \pm 9.7$)$ years. Sixty-six community health workers (62.9\%) had worked on the job for three years or less. Longer time on the job was positively associated with increased understanding of the disease and activities actually performed in TB control. Still, numerous flaws were observed in TB knowledge and control measures among community health workers. Improvements in continuing education for these workers could foster a more effective contribution to detecting new TB cases in the community and improved treatment adherence by patients.
\end{abstract}

Attitude of Health Personnel; Tuberculosis; Knowledge

\author{
Ethel Leonor Noia Maciel 1 \\ Rafael da Cruz Araújo Vieira 1 \\ Eliani Carrara Milani 1 \\ Mário Brasil 1 \\ Geisa Fregona 1 \\ Reynaldo Dietze 1
}

\section{Introdução}

Passada mais de uma década de sua declaração como um problema emergencial de saúde 1 , a tuberculose permanece sendo, entre as doenças infecciosas, a principal causa de óbitos entre adultos ao redor do mundo ${ }^{2}$. As estimativas mais recentes da Organização Mundial da Saúde (OMS) apontam o surgimento de 8,8 milhões de novos casos e 1,6 milhão de mortes por tuberculose. Para o Brasil, a cada ano surgirão $111 \mathrm{mil}$ novos casos 3 , dos quais 85 mil são notificados ao Sistema de Informações de Agravos de Notificação (SINAN), perfazendo uma taxa de incidência de 47/100 mil habitantes 4 . Tendo em vista a situação calamitosa do (des)controle da tuberculose no país, ao longo das décadas anteriores, o Conselho Nacional de Saúde (CNS), por meio da Resolução $n^{o} .284$ de 6 de agosto 1998, definiu a tuberculose como um problema prioritário de saúde no Brasil e estabeleceu diretrizes gerais de ação e metas para o Programa Nacional de Controle de Tuberculose (PNCT) 5 .

Seguindo a diretriz do PNCT de "horizontalização" do combate à tuberculose, por meio da expansão de suas atividades para todos os serviços de saúde do Sistema Único de Saúde (SUS), todos os planos nacionais e consensos para o controle da tuberculose que se seguiram enfatizaram a sua integração à atenção básica utilizando-se a Estratégia Saúde da Família e, em específico, o Programa de Agentes Comunitários de Saúde (PACS), 
como forma de ampliar o acesso ao diagnóstico e ao tratamento da tuberculose em todo o Brasil 6 .

Desde 1978, ano em que se realizou em Alma Ata, antiga União Soviética, a Conferência Internacional sobre Atenção Primária à Saúde, gerou-se grande expectativa em torno do trabalho dos agentes comunitários de saúde como atores nesta estratégia de extensão de cobertura, sendo declarado explicitamente que "ao nivel inicial de contato entre os indivíduos e o sistema de serviços de saúde, os cuidados primários são proporcionados por agentes de saúde da comunidade trabalhando em equipe" (Declaração de Alma Ata, 1979, apud Giffin \& Shiraiwa 7, p. 50).

Na Estratégia Saúde da Família, portanto, espera-se que o agente comunitário de saúde assuma um posto muitas vezes central, pois além de residir na comunidade em que atua, mostra-se familiarizado com seus valores, costumes e linguagem, podendo assim produzir uma união entre o uso de tecnologia/conhecimento em saúde e as crenças locais. O agente comunitário de saúde seria, então, um facilitador, capaz de construir pontes entre os serviços de saúde e a comunidade, identificando prontamente seus problemas, atuando no trabalho de prevenção de doenças e promoção da saúde 7,8,9.

No controle da tuberculose, espera-se basicamente que esse profissional seja capaz de identificar na comunidade, por meio de visitas domiciliares, aqueles indivíduos que apresentem tosse por três semanas ou mais (sintomático respiratório) e encaminhá-los aos serviços de saúde para pesquisa de tuberculose (busca ativa). Além disso, que orientem a família e a comunidade, acompanhem a tomada dos medicamentos pelos pacientes (tratamento supervisionado) e organizem reuniões com os membros da comunidade 10 .

Apesar de definitivamente incorporado ao controle de tuberculose em diversas partes do mundo, em especial onde o tratamento diretamente observado apresenta alta cobertura 3 , pouco se conhece da atuação do agente comunitário de saúde no PNCT de nosso país. Em vista disso, este estudo busca analisar os conhecimentos sobre as ações desenvolvidas pelos agentes comunitários de saúde no controle da tuberculose e a percepção que estes possuem do seu nível de conhecimento e de sua importância no enfrentamento da doença, no Município de Vitória, Espírito Santo.

\section{Metodologia}

Tratou-se de um estudo descritivo de corte transversal. A população base foi composta pe- los 370 agentes comunitários de saúde atuantes nas 24 Unidades de Saúde da Família do Município de Vitória, em março de 2005. Utilizou-se o programa Epi Info versão 3.3.2 (Centers for Disease Control and Prevention, Atlanta, Estados Unidos) para realizar o cálculo, e para não se arbitrar nenhum valor utilizou-se o valor máximo de prevalência de conhecimento sobre as ações para o controle de tuberculose esperada de $50 \%$, maximizando-se assim o tamanho da amostra esperada, obtendo-se o quantitativo de 107 agentes comunitários de saúde para esta população base.

Como as unidades estão divididas por seis (6) regiões de saúde, foi realizada uma amostragem aleatória por conglomerado. Cada região foi considerada como um conglomerado, sendo sorteadas dentro de cada um as unidades onde foram feitas as entrevistas. Utilizando-se o programa Microsoft Excel (Microsoft Corp., Estados Unidos) foi realizado um sorteio aleatório, levando em consideração o plano amostral de pelo menos uma unidade de saúde por região. Dessa forma, foi escolhida randomicamente a partir da seleção final sete Unidades de Saúde da Família que perfizeram uma total de 125 agentes comunitários de saúde atuantes.

A coleta dos dados foi realizada utilizando-se um questionário semi-estruturado, auto-aplicável e pré-testado fornecido a todos os agentes comunitários de saúde das Unidades de Saúde da Família selecionadas para obter as informações de interesse do estudo. Tal questionário era composto por perguntas fechadas e abertas e foi elaborado com base na cartilha Tuberculose: Informações para Agentes Comunitários de Saúde 11, no Manual Técnico para o Controle da Tuberculose: Cadernos de Atenção Básica 12 e no Controle da Tuberculose: Uma Proposta de Integração Ensino-Serviço 10, que trazem informações básicas para o agente comunitário de saúde sobre a doença e suas atribuições. Para as respostas discursivas, a análise da correção destas deu-se de maneira objetiva, sendo definidas como certas ou erradas conforme a presença ou não de palavras ou expressões-chave, núcleo do conhecimento avaliado. Esse instrumento foi validado em uma unidade de saúde da região de Maruípe com seis agentes comunitários de saúde, e as repostas obtidas não diferiram dos resultados encontrados por este estudo em relação as três categorias nas quais o instrumento foi dividido: (i) conhecimentos do agente comunitário de saúde acerca dos aspectos clínicoepidemiológicos da tuberculose; (ii) informações sobre as atividades realizadas pelo agente comunitário de saúde no controle da tuberculose e (iii) percepção do agente comunitário de 
saúde quanto ao seu nível de conhecimento e sua importância no controle da tuberculose.

Os dados obtidos por meio dos questionários foram transferidos para a planilha eletrônica Microsoft Excel. As análises estatísticas foram conduzidas no pacote estatístico Stata 9 (Stata Corp., College Station, Estados Unidos). Realizou-se o cálculo da média e desvio-padrão da idade e do tempo de trabalho dos agentes comunitários de saúde e das proporções das demais variáveis. A fim de investigar a influência do tempo de serviço como agente comunitário de saúde sobre as respostas dadas, a amostra foi estratificada de acordo com a mediana em dois grupos: (a) agentes comunitários de saúde com três anos ou menos de serviço e outro (b) com quatro anos ou mais de serviço. Na avaliação comparativa das variáveis estudadas empregou-se o teste de associação do qui-quadrado com significância menor que 0,05 .

O projeto foi previamente autorizado pela Secretaria Municipal de Saúde de Vitória e recebeu aprovação do Comitê de Ética em Pesquisa do Centro de Ciências da Saúde da Universidade Federal do Espírito Santo, de acordo com o que é estabelecido pela Resolução $n^{\circ}$. 196. Todos os participantes que aceitaram contribuir com o estudo assinaram o Termo de Consentimento Livre e Esclarecido, e foi garantido sigilo das informações. Nesse sentido, para manter a confidencialidade do estudo, optou-se por não revelar o nome das unidades selecionadas, uma vez que, todos os agentes comunitários de saúde lotados nestas unidades amostrais concordaram em participar do estudo e responderam ao instrumento.

\section{Resultados}

Vitória, capital do Espírito Santo, habilitado na Gestão Plena da Atenção Básica em janeiro de 1998, conta hoje com 28 unidades de saúde; destas, 24 Unidades de Saúde da Família e outros seis centros de referência (Centro de Especialidades Odontológicas, Centro de Referência em DST/AIDS, Centro de Atendimento ao Idoso, Centro de Controle de Zoonoses, Centro Municipal de Especialidades e Centro de Tratamento de Toxicômanos), destinados a atender a uma população projetada para 2007 de 320.824 habitantes, segundo o Instituto Brasileiro de Geografia e Estatística (IBGE, http://www.ibge.gov.br).

Ainda segundo o IBGE, apesar de possuir uma das mais altas rendas per capita do país e da bem estruturada rede de serviços básicos à saúde, a tuberculose permanece sendo um sério problema de saúde em Vitória. Em 2005, ano da coleta de dados desta pesquisa, foram notifica- dos 184 novos casos, produzindo uma incidência de 60,15/100 mil habitantes. Pelo elevado número de novos casos e pela alta incidência, o Município de Vitória foi incluído pelo PNCT entre os prioritários para o controle da doença ${ }^{13}$.

Com base no processo de seleção empregado, foram selecionadas sete Unidades de Saúde da Família onde estavam cadastrados 125 agentes comunitários de saúde, 33,8\% do universo de 370 agentes comunitários de saúde que atuavam em Vitória. No momento da coleta de dados, 20 agentes encontravam-se em férias ou em licença médica, não comparecendo à unidade de saúde. Assim, a amostra foi composta por 105 agentes comunitários de saúde, $28,38 \%$ da população, que após a assinatura do Termo de Consentimento Livre e Esclarecido responderam ao questionário fornecido.

Desses, a idade média e desvio-padrão foi de $34,5( \pm 9,7)$ anos, variando de 18 a 63 anos. Quanto ao tempo em que trabalha como agente comunitário de saúde, obteve-se uma média de 3,1 $( \pm 1,8)$ anos, sendo que o maior período foi de sete anos e o menor, referente a um recém-admitido, inferior a um mês. Dos 105 agentes comunitários de saúde entrevistados, 66 (62,9\%) atuavam nesta profissão há um período igual ou inferior a três anos, ao passo que 37,1\% (39/105) há quatro anos ou mais.

As variáveis incluídas no questionário foram agrupadas em três blocos temáticos, a saber: (i) conhecimentos do agente comunitário de saúde a cerca dos aspectos clínico-epidemiológicos da tuberculose (Tabela 1); (ii) informações sobre as atividades realizadas pelo agente comunitário de saúde no controle da tuberculose (Tabela 2); e (iii) percepção do agente comunitário de saúde quanto ao seu nível de conhecimento e sua importância no controle da tuberculose (Tabela 3).

Quando avaliado o conhecimento que o agente comunitário de saúde possui dos principais sintomas da tuberculose, verificou-se que a febre foi aquele mais presente nas respostas apresentadas, com 62,9\% (66/105). A tosse por mais de três semanas, reconhecidamente o sintoma mais característico da tuberculose e fundamental para a identificação dos sintomáticos respiratórios, foi o relatado com menor freqüência sendo corretamente assinalado por $26,7 \%$ (28/105) dos agentes comunitários de saúde. Observou-se que parte dos agentes respondeu "tosse", porém, sem referência ao período de duração. Essa resposta não foi considerada correta uma vez que o fator tempo é fundamental para a caracterização do sintoma. O emagrecimento foi identificado como sintoma de tuberculose por 35/105 $(33,3)$ dos agentes comunitários de saúde. Apenas 12/105 
Conhecimentos do agente comunitário de saúde acerca dos aspectos clínico-epidemiológicos da tuberculose.

\begin{tabular}{|c|c|c|c|c|c|}
\hline & \multicolumn{4}{|c|}{ Tempo de atuação como agente comunitário de saúde } & \multirow[t]{3}{*}{$\mathbf{p}$} \\
\hline & \multicolumn{2}{|c|}{3 anos ou menos $(n=66)$} & \multicolumn{2}{|c|}{4 anos ou mais $(n=39)$} & \\
\hline & $\mathbf{n}$ & $\%$ & $\mathbf{n}$ & $\%$ & \\
\hline \multicolumn{6}{|l|}{ Sintomas da tuberculose * } \\
\hline Tosse por mais de 3 semanas & 17 & 25,8 & 11 & 28,2 & 0,822 \\
\hline Febre & 40 & 60,6 & 26 & 66,7 & 0,676 \\
\hline Emagrecimento & 14 & 21,2 & 21 & 53,8 & $0,001 * \star \star$ \\
\hline Formas de transmissão da tuberculose ** & 29 & 43,9 & 21 & 53,8 & 0,419 \\
\hline Sintomático respiratório ** & 1 & 1,5 & 2 & 5,1 & 0,554 \\
\hline \multicolumn{6}{|l|}{ Exames diagnósticos para a tuberculose * } \\
\hline Escarro & 48 & 72,7 & 36 & 92,3 & 0,022 *** \\
\hline Raio-X & 12 & 18,2 & 14 & 35,9 & 0,061 \\
\hline PPD & 0 & 0,0 & 1 & 2,6 & 0,371 \\
\hline Tratamento supervisionado ** & 24 & 36,4 & 28 & 71,8 & $0,001 * \star \star$ \\
\hline \multicolumn{6}{|l|}{ Medicações } \\
\hline Conhece ao menos uma & 4 & 6,1 & 12 & 30,8 & $0,001 * \star \star$ \\
\hline
\end{tabular}

* Mais de uma resposta permitida;

** Responderam corretamente;

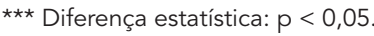

Tabela 2

Atividades realizadas pelo agente comunitário de saúde no controle da tuberculose.

Tempo de atuação como agente comunitário de saúde

3 anos ou menos $(n=66$ )

4 anos ou mais $(n=39)$

$\%$

$\%$

Orientação ao paciente de tuberculose *

Ao menos duas orientações

24

36,4

Ao menos uma orientação

45

68,2

29

74,4

$0,000 * \star *$

Conduta com o familiar *

Ao menos uma conduta realizada

Escarro

12,1

62,5

Raio-X

25,0

PPD

Explicação sobre contágio

37,5

12,5

Identificação de caso de tuberculose **

Tosse

33,3

Emagrecimento

40,0

36

92,3

$0,004 * * *$

Mais de uma resposta permitida;

** Responderam afirmativamente;

*** Diferença estatística: $p<0,05$. 
$(11,4 \%)$ foram capazes de relacionar todos os três sintomas.

Já em relação à forma de transmissão do Mycobacterium tuberculosis, agente etiológico da tuberculose, menos da metade dos agentes comunitários de saúde $(47,6 \%$; 50/105) possuíam o conhecimento de que sua veiculação se dava através do ar, a partir da tosse, fala ou espirro de um indivíduo doente. Um percentual ainda menor foi obtido na compreensão do termo "sintomático respiratório", em que apenas 3/105 $(2,9 \%)$ responderam corretamente a este questionamento.

Do conjunto de agentes comunitários de saúde, 84 (80\%) mostraram saber que a principal forma de diagnóstico da tuberculose se faz por meio de exame do escarro. O raio-X foi identificado em 26 (24,8\%) das respostas dadas. Quanto à "supervisão do tratamento de tuberculose", cerca da metade dos agentes comunitários de saúde (52/105; 49,5\%) demonstrou conhecer esta atividade. Contudo, apenas 16/105 (15,2\%) souberam relatar ao menos um dos três medicamentos usualmente utilizados no tratamento da doença.

Na Tabela 1 são comparados o nível de conhecimento dos agentes comunitários de saúde frente ao tempo em este desenvolve esta função. Observa-se que o tempo de atividade mostra-se um fator importante na proporção de respostas corretas para algumas questões. Dessa forma, diferenças significativas $(\mathrm{p}<0,05)$ foram observadas no reconhecimento do emagrecimento como sintoma da tuberculose, do exame de escarro como método diagnóstico, do conceito de "tratamento supervisionado" e no conhecimento de, ao menos, uma medicação utilizada no tratamento dos pacientes. Para todos esses, um maior período exercendo essa atividade encontra-se associado com um nível de compreensão em torno da doença mais elevado.

Para analisar as atividades efetivamente realizadas no controle da tuberculose foram formuladas três questões. Na primeira solicitou-se ao agente comunitário de saúde que citasse quais as orientações que são usualmente oferecidas aos pacientes com tuberculose. Uma vez que as questões permitiam respostas discursivas, foram avaliadas quais se mostravam corretas e quantificadas, sem produzir escala de importância. Entre as instruções relatadas, que possuem descrições nos manuais oficiais de controle da tuberculose utilizados como parâmetros, podemos citar: tomada das medicações corretamente; não abandonar o tratamento; evitar bebidas alcoólicas e fumo durante o tratamento; manter o ambiente bem arejado; fazer uma boa alimentação; ingerir bastante líquido; procurar a unidade de saúde se sentir algum efeito colateral relacionado à toma- da dos medicamentos; e a falta de necessidade de se separar utensílios. Dessa maneira, destaca-se que 24/105 (22,9\%) não foram capazes de relatar ao menos uma orientação, ao passo que 50,5\% (53/105) citaram duas ou mais instruções rotineiramente oferecidas aos pacientes diagnosticados com tuberculose.

Quando analisada a conduta tomada diante dos familiares e contactantes dos casos de tuberculose acompanhados, 32 dos 105 (30,5\%) souberam relatar uma ou mais a serem seguidas, conforme os manuais oficiais. A identificação de novos casos de tuberculose, uma das atividades fundamentais do agente comunitário de saúde no controle da tuberculose, foi relatada por cerca de um quarto dos entrevistados $(24,8 \% ; 26 / 105)$.

Destaca-se a influência do tempo de serviço como agente comunitário de saúde sobre as ações desenvolvidas por este no controle da doença, com um aumento significativo $(p<0,05)$ na capacidade de oferecer orientações ao paciente com tuberculose sobre a doença, bem como incremento no número de instruções e condutas que são passadas aos contactantes dos casos. Porém não houve diferenças estatísticas $(\mathrm{p}<0,05)$ entre as proporções específicas de cada conduta nem entre a identificação de casos de tuberculose em cada grupo.

Por fim, com a Tabela 3, busca-se analisar a percepção que os agentes comunitários de saúde possuem tanto de seu nível de conhecimento em relação à doença quanto a importância que ele e suas atividades possuem no efetivo controle da tuberculose. Diferenças significativas são encontradas nos níveis intermediários de conhecimento, em que se observa que os agentes comunitários de saúde com maior tempo de atuação auto-avaliam seu conhecimento como bom em uma proporção superior à dos que trabalham a menos tempo $(\mathrm{p}<0,05)$. Já no nível $r e$ gular ocorre uma inversão, predominando nesta faixa os agentes comunitários de saúde com três ou menos anos de serviço. Independentemente disso, 97,1\% (102/105) dos agentes comunitários de saúde consideram que as atividades desenvolvidas por eles são de grande importância no controle da tuberculose.

\section{Discussão}

Ainda que a literatura seja restrita, tem-se observado um interesse crescente pelo papel e pelas atividades atribuídas aos agentes comunitários de saúde atuantes no Brasil. Uma recente revisão traz que cerca de $60 \%$ da produção bibliográfica sobre o tema concentra-se nos anos posteriores a 2000 14. Em nosso país, a primeira experiência 
Percepção do agente comunitário de saúde quanto ao seu nível de conhecimento e sua importância no controle da tuberculose.

\begin{tabular}{|c|c|c|c|c|c|}
\hline & \multicolumn{4}{|c|}{ Tempo de atuação como agente comunitário de saúde } & \multirow[t]{3}{*}{ p } \\
\hline & \multicolumn{2}{|c|}{3 anos ou menos $(n=66)$} & \multicolumn{2}{|c|}{4 anos ou mais $(n=39)$} & \\
\hline & n & $\%$ & $\mathbf{n}$ & $\%$ & \\
\hline \multicolumn{6}{|l|}{ Nível de conhecimento } \\
\hline Ótimo & 3 & 4,50 & 4 & 10,30 & 0,420 \\
\hline Bom & 23 & 34,80 & 22 & 56,40 & $0,041 * \star$ \\
\hline Regular & 37 & 56,10 & 11 & 28,20 & $0,008 * \star$ \\
\hline Ruim & 2 & 3,03 & 2 & 5,13 & 0,627 \\
\hline Importância para o controle * & 63 & 95,45 & 39 & 100,00 & 0,293 \\
\hline
\end{tabular}

* Responderam afirmativamente;

** Diferença estatística: $p<0,05$

relatada no sentido de utilizar agentes de saúde nos moldes propostos pela Conferência Internacional sobre Atenção Primária à Saúde se deu no Rio de Janeiro no ano de 1986, onde moradoras de favelas foram capacitadas, supervisionadas e remuneradas para desempenhar, em suas comunidades, ações básicas de saúde, em uma extensão de cobertura do Programa de Assistência Integral à Saúde da Mulher e da Criança 9 .

Tal qual em nosso estudo, a revisão mostra ainda que a metodologia mais empregada nos trabalhos publicados foi a experimental, fazendo uso de coleta de dados, grupos de comparação, estudos de acompanhamento ou estudos de casos. Contudo, não se encontrou estudos que buscassem avaliar o grau de conhecimento dos agentes comunitários de saúde em torno de um programa de saúde específico no qual este esteja inserido. Ainda que incorporados a diversos programas de saúde, como o combate à dengue, a saúde do idoso e a saúde maternoinfantil, e desempenhando com eficiência uma série de atividades, em muitas outras circunstâncias os resultados não têm sido promissores. Sugere-se que isso se deva tanto à excessiva carga de atividades que estes recebem quanto a deficiências nos processos de seleção, treinamento e supervisão ${ }^{15}$. Os achados deste estudo também apontam para a possibilidade de que as oportunidades inicialmente vislumbradas no processo de integração dos agentes comunitários de saúde ao controle da tuberculose não estejam se concretizando.

A realização da busca ativa de sintomáticos respiratórios para diagnóstico precoce de casos de tuberculose é uma das tarefas a ser realizada pelo agente comunitário de saúde nesta nova proposta de descentralização do PNCT. A fim de avaliar a incorporação dessa atividade, um estudo analisou a percepção dos enfermeiros supervisores do PACS de um distrito de saúde do Município de Ribeirão Preto, Estado de São Paulo. Da investigação dos discursos produzidos, debilidades referentes ao processo de conformação e organização do PACS foram levantadas. Dessas destacamos a capacitação insuficiente dos agentes 16 . Tal constatação revela-se em nossos achados. De maneira geral, os agentes comunitários de saúde possuíam um conhecimento muito limitado da sintomatologia clássica da tuberculose, em especial no que se refere à tosse prolongada e, por conseguinte, quanto à definição de um sintomático respiratório. Apenas 3 dos 105 agentes comunitários de saúde souberam apresentar uma descrição correta do termo. Essa observação contrasta com o dado de que um quarto dos entrevistados (26/105) já havia identificado um novo caso de tuberculose. A consistência desse dado torna-se questionável quando avaliado que, de maneira geral, mesmo não entendendo a tosse prolongada como um sintoma importante, 20 das 26 identificações relatadas se deu por meio desta característica.

Chama a atenção o fato de o principal sintoma apontado pelos agentes comunitários de saúde ter sido a febre. Pela análise da cartilha distribuída aos agentes comunitários de saúde 11, esse sintoma é o que recebe menor destaque. A tosse e a falta de apetite/emagrecimento, além de explicitadas no texto, recebem ilustrações. Soma-se a isso o fato de esse ser o sintoma menos aparente da tuberculose. Além de indicar a falta de um treinamento específico sobre a doença, essas distorções nas informações apresen- 
tadas apontam a necessidade de investigações do processo de formação deste conhecimento.

No que tange ao conhecimento do processo de disseminação da tuberculose, os agentes comunitários de saúde entrevistados revelaram um nível de entendimento igualmente baixo. Apenas cerca da metade dos agentes comunitários de saúde foi capaz de oferecer uma resposta correta para essa questão. Em um estudo realizado na província de Tigray, Norte da Etiópia, que buscava avaliar os conhecimentos e o despreparo de agentes comunitários de saúde voluntários na supervisão de tratamentos de tuberculose pulmonar em uma região rural, registrou que 80,3\% dos 279 agentes comunitários de saúde entrevistados foram capazes de reconhecer a tosse como principal forma de transmissão da doença, portanto superior ao observado entre os agentes comunitários de saúde entrevistados 17 . Do estudo em Tigray sugere-se que as falhas no entendimento das causas e da transmissão da tuberculose podem levar a uma baixa adesão dos paciente ao tratamento, em especial pelas dificuldades que surgem da interação entre o cuidador e o doente.

Essa lacuna no conhecimento acerca da transmissão do M. tuberculosis possui implicações para além da orientação correta de pacientes e contactantes. Uma vez que, dentre suas atividades encontram-se a visita domiciliar e a supervisão da tomada da medicação, o agente comunitário de saúde torna-se, como os demais profissionais de saúde, exposto a um risco de infecção pelo bacilo superior ao da população em geral. Sabidamente, o contato com pacientes bacilíferos é o principal fator de risco para a infecção, evidenciado pela conversão tuberculínica 12. Essa percepção é confirmada em uma revisão sistemática da literatura sobre a tuberculose entre profissionais de saúde de países com baixa e média rendas, onde se observa que a tuberculose é um sério problema de saúde ocupacional entre eles 18 . Ainda que na literatura esse risco não tenha sido quantificado entre agentes comunitários de saúde, a falta de conhecimento acerca da disseminação do agente infeccioso pode estar envolvida com a redução da sua capacidade de proteger-se de maneira adequada, intensificando este risco.

Deficiências importantes também foram observadas quanto ao entendimento acerca do tratamento supervisionado e da medicação utilizada. Por outro lado, o conhecimento sobre o diagnóstico revelou-se elevado. Pela avaliação do material educacional utilizado pelos agentes comunitários de saúde, não é possível compreender a distorção entre o conhecimento sobre cada área, uma vez que distinções aparentes não são feitas nas cartilhas. É possível, porém, que tal privilégio à detecção e não ao acompanhamento do tratamento se desenvolva na orientação dos agentes comunitários de saúde pelos seus supervisores nas unidades de saúde, devendo este processo de aprendizado ser alvo de investigação.

Pode ser observado por meio das respostas dadas ao questionário, tanto em relação às condutas a serem tomadas e orientações a serem dadas diante de um caso de tuberculose e aos seus contactantes, uma dificuldade no entendimento de ações necessárias ao manejo do caso. Esse achado contrasta com o nível sensivelmente superior de conhecimento acerca dos aspectos clínico-epidemiológicos da tuberculose. Resultado semelhante pode ser observado em estudo realizado no Iraque, onde os profissionais de saúde, entre os quais se incluíam os agentes comunitários de saúde, também demonstraram elevado conhecimento sobre a doença, mas grandes dificuldades no manejo do paciente suspeito de tuberculose 19. Também nesse sentido, a elevada proporção de casos de tuberculose falso-positivos encontrada em trabalho realizado em Burkina Faso indica um melhor treinamento desses profissionais 20 . Disso sugere-se a necessidade de investimento não apenas em formação didática, mas também em um constante monitoramento das atividades por eles realizadas, no intuito de identificar suas fragilidades.

É importante também ressaltar que outra pesquisa realizada em Vitória por Maciel et al. 21 identificou que os enfermeiros e médicos possuem conhecimento suficiente sobre a tuberculose, no entanto, os resultados apontaram que não é o conhecimento, mas outros fatores os responsáveis pela manutenção desses índices da doença em nossa região. Esses resultados discordantes entre os dois estudos apontam para diferenças existentes entre as categorias profissionais e parecem indicar a pouca socialização do conhecimento na equipe multidisciplinar.

Um dos pontos que podem ser levantados é que, uma vez que o paciente chega ao serviço ele possui o atendimento necessário à sua demanda, talvez uma das principais dificuldades seja ainda a identificação destes pacientes de forma precoce na comunidade e seu acesso aos serviços de maneira adequada. Nosso estudo indica que a efetiva implantação dessa estratégia de busca ativa dos casos perpassa pelo investimento em treinamento dos agentes comunitários de saúde, que possuem um papel fundamental no controle da tuberculose.

Faz-se necessário, no entanto, apontar que as limitações do nosso estudo se referem principalmente à natureza do uso dos inquéritos do tipo "conhecimento, atitudes e percepções em 
saúde", pois como não realizamos a observação e avaliação efetiva da rotina dos agentes, nosso instrumento investigou apenas as dimensões cognitivas e valorativas dos agentes comunitários de saúde em relação ao controle da tuberculose e, portanto, as conclusões obtidas por este estudo refletem os enunciados dos entrevistados sobre suas ações, mas não podem descrever as atividades efetivamente desenvolvidas no cotidiano das unidades de saúde pelos agentes comunitários. Outro ponto a ser destacado é que em algumas perguntas houve um número alto de respostas em branco, o que pode ter influenciado a análise final com uma maior probabilidade de falhar em rejeitar a hipótese de que o tempo não tem influência sobre o conhecimento do agente comunitário de saúde, quando na verdade, esta hipótese deveria se rejeitada (erro do tipo II).

A despeito, porém, do nível de conhecimento apresentado por meio dos questionários, os agentes comunitários de saúde, em sua quase totalidade, acreditam que seu papel e suas atividades são importantes para o controle da tuberculose. Contudo, estes possuem uma percepção realista do seu nível de conhecimento acerca da doença e suas atividades. Um achado interessante é a influência produzida pelo tempo que o agente comunitário de saúde exerce suas atividades e a resposta dada quanto ao nível de conhecimento que possui. Maior proporção de agente comunitário de saúde com quatro ou mais anos de exercício nesta atividade avaliaram seu conhecimento como bom, ao passo que a maior parte daqueles com menos de quatro anos perceberam seu conhecimento como apenas regular, apresentando diferenças estatisticamente significantes. Em diversas outras questões o tempo de emprego revelou-se um importante definidor do nível de conhecimentos e das práticas realizadas, o que encontra subsídios no fato destes acumularem experiências no contato com os demais componentes da equipe de saúde e com a população, além da maior probabilidade de terem passado por treinamentos. No levantamento dos conhecimentos e das práticas dos profissionais de saúde realizado no Iraque, o conhecimento sobre a tuberculose esteve associado significativamente com a idade e o tempo de serviço 19 .

\section{Considerações finais}

Da apreciação do conjunto dos resultados obtidos, entendemos que os agentes comunitários de saúde do Município de Vitória, envolvidos no PNCT, podem não estar alcançando os resultados esperados na contribuição para um aumento importante da detecção de novos casos na comu- nidade nem para maior adesão dos pacientes ao tratamento.

Segundo o relatório do Departamento de Recursos Humanos para a Saúde da OMS 22, que avalia a situação dos programas de saúde que incorporaram o agente comunitário em seus organogramas, suas atividades, seu custo e o impacto sobre o estado de saúde da comunidade onde atuam, pode-se reconhecer nele um ator fundamental no processo de expansão da cobertura e do acesso aos serviços de saúde, podendo por meio de suas atividades levar a uma melhora nos desfechos de saúde. Ressalta, porém, que para obter êxito em larga escala os programas de agentes comunitários de saúde devem ser cuidadosamente planejados, os recursos financeiros assegurados, além da vontade política e do apoio da comunidade. Já o agente comunitário de saúde, para que seja capaz de desempenhar com sucesso suas tarefas, necessita de treinamento e supervisão regular além de uma logística de apoio confiável 22 .

De fato, quando esses fatores encontramse disponíveis, exemplos de sucesso podem ser observados como em Bangladesh, onde altas taxas de detecção e tratamentos concluídos foram atingidos por meio desses profissionais baseados na comunidade 23 . Conclusão semelhante pôde ser obtida na Cidade do Cabo, África do Sul, onde o trabalho dos agentes comunitários de saúde contribuiu de maneira superior para a melhora do programa de controle da tuberculose quando comparado com a abordagem que fazia uso exclusivo das unidades de saúde 24 .

Outra questão levantada neste estudo, agora no campo da ética e que deve ser alvo de intensa reflexão, trata da exposição de profissionais, com evidente carência de entendimento sobre a transmissão da tuberculose, a riscos de infecção sem a orientação devida e a provisão de equipamentos de proteção. Nesse sentido, torna-se fundamental a compreensão do agente comunitário de saúde sobre esses riscos, dos métodos de proteção e de um acompanhamento constante de sua condição de saúde.

Observa-se ainda, a importância que o tempo possui na formação do agente comunitário de saúde e no bom desempenho de suas atividades. Torna-se compreensível, portanto, que todos os pré-requisitos levantados para o bom andamento dos programas de saúde que incorporaram os agentes comunitários de saúde convirjam para a manutenção de pessoas bem selecionadas e remuneradas, realizando atividades compatíveis com seu nível educacional, que recebem treinamento constante e supervisão adequada.

Em relação ao processo de supervisão dos agentes comunitários de saúde, um estudo rea- 
lizado em Natal, Estado do Rio Grande do Norte, Brasil, indicou que este ainda se encontra permeado por mecanismos de controle, principalmente quando se acha relacionado à concepção de supervisão de cada enfermeiro 25. Portanto, é cada vez mais urgente o desenvolvimento de instrumentos gerenciais e de trabalho que sejam dotados de um caráter pedagógico. É preciso perceber que a visão fragmentada existente nos processos educativos, assim como a prática profissional pouco cooperativa, bem como o distanciamento entre as diversas disciplinas contribuem para a manutenção da fragmentação no cotidiano dos serviços 26 .

Espera-se, diante dos resultados encontrados pela nossa pesquisa, desencorajadores a princípio, que possam nortear ações para a melhoria da qualidade dos serviços desenvolvidos pelos agentes comunitários de saúde nos programas de controle da tuberculose e com isso auxiliar a consolidação do Programa Saúde da Família em nossa região. Esta, somente se efetivará com a construção de um projeto assistencial comum com complementaridade dos trabalhos especializados, em que todos os profissionais envolvidos estabeleçam interação entre si e com os usuários.

Por fim cabe ressaltar para que essa integralidade realmente ocorra algumas questões devem tornar-se alvo de discussão, pois uma vez regulamentada a profissão de agente comunitário de saúde 27 , algumas questões devem tornar-se neste momento prioritárias, quais sejam, a avaliação do processo de contratação do SUS, os planos de carreira e principalmente os processos de educação permanente que sem dúvida se tornam o eixo reorientador para a consolidação desta nova política no país.

\section{Resumo}

Este estudo analisa o conhecimento dos agentes comunitários de saúde no controle da tuberculose e a autopercepção do seu nível de conhecimento e de sua importância no enfrentamento da doença, no Município de Vitória, Espírito Santo, Brasil. Tratou-se de um estudo de corte transversal. Um questionário semi-estruturado, auto-aplicável e pré-testado foi preenchido por 105 agentes comunitários de saúde randomicamente selecionados. A comparação das proporções entre os grupos formados pela estratificação por tempo de serviço foi realizada usando-se o teste qui-quadrado com nível de significância de 5\%. A idade média foi de 34,5 $( \pm 9,7)$ anos. Atuavam há três anos ou menos 66 agentes comunitários de saúde (62,9\%). Observou-se que um maior tempo de atividade está relacionado com um aumento do nível de compreensão em torno da doença, bem como das atividades efetivamente realizadas no controle da tuberculose. Contudo, de maneira geral, os conhecimentos e as ações do agente comunitário de saúde mostraram-se muito falhos. Entende-se que com melhorias na educação permanente desses profissionais seria possível uma maior contribuição deles para o aumento da detecção de novos casos na comunidade e para maior adesão dos pacientes ao tratamento.

Atitude do Pessoal de Saúde; Tuberculose; Conhecimento

\section{Colaboradores}

E. C. Milani e M. Brasil participaram da revisão de literatura, elaboração da metodologia, análise dos resultados e aprovação da versão final. R. C. A. Vieira e G. Fregona colaboraram na concepção do projeto de pesquisa, análise e interpretação dos dados e redação. R. Dietze e E. L. N. Maciel participaram de todas as etapas da pesquisa e da revisão crítica. 


\section{Referências}

1. World Health Organization. TB: a global emergency. Geneva: World Health Organization; 1993.

2. Dye C, Scheele S, Dolin P, Pathania V, Raviglione MC. Consensus statement. Global burden of tuberculosis: estimated incidence, prevalence, and mortality by country. WHO Global Surveillance and Monitoring Project. JAMA 1999; 282:677-86.

3. World Health Organization. Global tuberculosis control: surveillance, planning, financing. Geneva: World Health Organization; 2007.

4. Secretaria de Vigilância em Saúde, Ministério da Saúde. Sistema nacional de vigilância em saúde: relatório de situação: Espírito Santo. 2a Ed. Brasília: Ministério da Saúde; 2006.

5. Ruffino-Netto A. Impacto da reforma do setor saúde sobre os serviços de tuberculose no Brasil. Bol Pneumol Sanit 1999; 7:7-18.

6. Castelo Filho A, Kritski AL, Barreto AW, Lemos ACM, Ruffino-Netto A, Guimarães CA, et al. II Consenso Brasileiro de Tuberculose: diretrizes brasileiras para tuberculose 2004. J Bras Pneumol 2004; 30 Suppl 1:S57-86.

7. Giffin K, Shiraiwa T. O agente comunitário de saúde pública: a proposta de Manguinhos. Cad Saúde Pública 1989; 5:24-44.

8. Haines A, Sanders D, Lehmann U, Rowe AK, Lawn JE, Jan S, et al. Achieving child survival goals: potential contribution of community health workers. Lancet 2007; 369:2121-31.

9. Nunes MO, Trad LB, Almeida BA, Homem CR, Melo MCIC. O agente comunitário de saúde: construção da identidade desse personagem híbrido e polifônico. Cad Saúde Pública 2002; 18:1639-46.

10. Fundação Nacional de Saúde, Ministério da Saúde/Centro de Referência Professor Hélio Fraga/ Sociedade Brasileira de Pneumologia e Tisiologia. Controle da tuberculose: uma proposta de integração ensino-serviço. 5a Ed. Brasília: Fundação Nacional de Saúde, Ministério da Saúde/Rio de Janeiro: Centro de Referência Professor Hélio Fraga/ Sociedade Brasileira de Pneumologia e Tisiologia; 2002.

11. Secretaria de Políticas de Saúde, Ministério da Saúde. Tuberculose: informações para agentes comunitários de saúde. Brasília: Ministério da Saúde; 2001.

12. Departamento de Atenção Básica, Secretaria de Políticas de Saúde, Ministério da Saúde. Manual técnico para o controle da tuberculose: cadernos de atenção básica. 6a Ed. Brasília: Ministério da Saúde; 2002.

13. Coordenação Geral de Doenças Endêmicas, Departamento de Vigilância Epidemiológica, Secretaria de Vigilância em Saúde, Ministério da Saúde. Programa nacional de controle da tuberculose. Brasília: Ministério da Saúde; 2002.

14. Kluthcovsky ACGC, Takayanagui AMM. Community health agent: a literature review. Rev Latinoam Enferm 2006; 14:957-63.
15. Witmer A, Seifer SD, Finocchio L, Leslie J, O’Neil EH. Community health workers: integral members of the health care work force. Am J Public Health 1995; 85:1055-8.

16. Muniz JN, Palha PF, Monroe AA, Gonzales RC, Ruffino-Netto A, Villa TCS. A incorporação da busca ativa de sintomáticos respiratórios para o controle da tuberculose na prática do agente comunitário de saúde. Ciênc Saúde Coletiva 2005; 10:315-21.

17. Mesfin MM, Tasew TW, Tareke IG, Richard MRJ. Community health workers: their knowledge on pulmonary tuberculosis and willingness to be treatment supervisors in Tigray, northern Ethiopia. Ethiopian Journal of Health Development 2005; 19:28-34.

18. Joshi R, Reingold AL, Menzies D, Pai M. Tuberculosis among health-care workers in low- and middleincome countries: a systematic review. PLoS Med 2006; 3:2376-91.

19. Hashim DS, Al Kubaisy W, Al Dulayme A. Knowledge, attitudes and practices survey among health care workers and tuberculosis patients in Iraq. East Mediterr Health J 2003; 9:718-31.

20. Simonnet F, Prazuck T. Do health workers recognise tuberculosis in Burkina Faso? Lancet 1988; 2:792.

21. Maciel ELN, Araújo WK, Giacomin SS, Jesus FA, Rodrigues PM, Dietze R. O conhecimento de enfermeiros e médicos que trabalham na Estratégia Saúde da Família acerca da tuberculose no Município de Vitória-ES: um estudo de corte transversal. Ciênc Saúde Coletiva; no prelo.

22. World Health Organization. Community health workers: what do we know about them? Policy briefs. Geneva: World Health Organization; 2007.

23. Chowdhury AM, Chowdhury S, Islam MN, Islam A, Vaughan JP. Control of tuberculosis by community health workers in Bangladesh. Lancet 1997; 350:169-72.

24. Dudley L, Azevedo V, Grant R, Schoeman JH, Dikweni L, Maher D. Evaluation of community contribution to tuberculosis control in Cape Town, South Africa. Int J Tuberc Lung Dis 2003; 7(9 Suppl 1):S48-55.

25. Araújo MBS, Villas Bôas LMFM, Timóteo RPS. A prática gerencial do enfermeiro no PSF na perspectiva da sua ação pedagógica educativa: uma breve reflexão. Ciênc Saúde Coletiva; no prelo.

26. Silva JA, Dalmaso ASW. O agente comunitário de saúde e suas atribuições: os desafios para os processos de formação de recursos humanos em saúde. Interface Comum Saúde Educ 2002; 6:75-83.

27. Levy FM, Matos PE, Tomita NE. Programa de agentes comunitários de saúde: a percepção de usuários e trabalhadores da saúde. Cad Saúde Pública 2004; 20:197-203.

Recebido em 12/Jun/2007

Versão final reapresentada em 20/Set/2007 Aprovado em 08/Nov/2007 